\title{
Primer reporte de miasis hospitalaria por Lucilia cuprina (Diptera: Calliphoridae) en Costa Rica
}

\author{
Lucía Quesada-Lobo ${ }^{1,2}$, Adriana Troyo3 ${ }^{3}$, Ólger Calderón-Arguedas ${ }^{3}$ \\ ${ }^{1}$ Programa de Posgrado en Parasitología, Facultad de Microbiología, Universidad de Costa Rica, San José, \\ Costa Rica \\ 2 Laboratorio Clínico, Hospital México, San José, Costa Rica \\ ${ }^{3}$ Departamento de Parasitología, Facultad de Microbiología, Universidad de Costa Rica, San José, Costa Rica \\ Institución donde se llevó a cabo el trabajo: Facultad de Microbiología, Universidad de Costa Rica, San José, \\ Costa Rica
}

Introducción. Las miasis hospitalarias son entidades con una importancia manifiesta en salud pública. La documentación de este tipo de casos es escasa en la literatura biomédica regional y mundial.

Objetivo. Informar un caso de miasis hospitalaria en Costa Rica, donde el agente etiológico implicado fue Lucilia cuprina (Diptera: Calliphoridae). Este caso de miasis hospitalaria figura como el primer informe para Latinoamérica asociado con este agente etiológico.

Presentación del caso. Una paciente de 91 años de edad, con signos de inmunosupresión, afectación grave de la función pulmonar y asistencia respiratoria mecánica, presentó larvas en ambas fosas nasales al séptimo día después del ingreso hospitalario. Varios ejemplares fueron recolectados y procesados para su identificación.

La identificación taxonómica de los ejemplares recolectados estableció que la especie de los muscomorfos correspondía a L. cuprina.

Conclusión. El presente constituye el primer caso de miasis hospitalaria por L. cuprina en la literatura biomédica de Costa Rica y el primero registrado en Latinoamérica.

Palabras clave: miasis, informes de casos, Costa Rica.

doi: http://dx.doi.org/10.7705/biomedica.v32i4.690

First report of nosocomial myiasis by Lucilia cuprina (Diptera: Calliphoridae) in Costa Rica

Introduction. Nosocomial myiases can be an important condition from a public health perspective. However, cases of this condition reported in regional and worldwide biomedical literature are scarce.

Objective. A case of nosocomial myiasis is reported from Costa Rica, where the species involved was Lucilia cuprina (Diptera: Calliphoridae).

Materials and methods. A 91-year-old patient with signs of immunosuppression, severe impairment of lung function, and mechanical ventilation presented larvae in both nostrils on the seventh day after admission. Five specimens were collected and processed for identification.

Results. The taxonomic identification of the specimens established that the muscoid fly species was L. cuprina.

Conclusion. This is the first case of nosocomial myiasis reported from Costa Rica and in Latin America for which the etiological agent is $L$. cuprina.

Key words: myiasis, case reports, Costa Rica.

doi: http://dx.doi.org/10.7705/biomedica.v32i4.690

\author{
Correspondencia: \\ Lucía Quesada-Lobo, Laboratorio Clínico, Hospital México, San \\ José, Costa Rica. \\ Teléfono: (506) 8385 5229; fax: (506) 25114360 \\ luquesadalobo@hotmail.com
}

Recibido: 23/03/12; aceptado:05/07/12

\section{Contribución de los autores:}

Lucía Quesada-Lobo realizó la documentación del caso, toma de muestras, revisión bibliográfica y fue la principal responsable de la redacción del texto. Adriana Troyo aportó en la identificación del material presentado, análisis crítico y revisión del documento. Ólger Calderón-Arguedas procesó, identificó y fotografió el material presentado, colaboró con la revisión bibliográfica, el análisis crítico y la redacción del documento.
Las miasis constituyen infestaciones de órganos y tejidos del ser humano y animales vertebrados, provocadas por larvas de mosca (1). Representan un fenómeno mundial, principalmente asociado con climas húmedos y cálidos (2). Existen diferentes términos para designar el tipo de miasis asociado con una determinada región anatómica afectada:

critico y la redacción del documento. 
miasis dérmica, cutánea o subdérmica; miasis urogenital; miasis gastrointestinal o entérica; miasis oftálmica u ocular; miasis nasofaríngea; etc. (2). Cuando las larvas se alojan superficialmente bajo la piel, el cuadro se denomina miasis progresiva; si lo que se produce es inflamación con marcado abultamiento, el término utilizado es miasis "foruncular"; y en el caso de que una herida o úlcera se infeste con larvas de moscas, se suele hacer referencia a una miasis traumática (2).

Las especies de mosca asociadas a las miasis pueden clasificarse como obligadas, facultativas o accidentales. En las miasis obligadas la larva es biontófaga, es decir, requiere de tejido vivo como sustrato alimenticio, al menos en alguna fase de su ciclo vital; Cochliomyia hominivorax y Dermatobia hominis son dos de las especies más conocidas como agentes etiológicos de miasis obligadas en los entornos neotropicales (2). Por el contrario, en las miasis facultativas o accidentales las larvas son necrobiotófagas, pues se asocian con cadáveres en descomposición o con tejido necrótico cuando infestan un organismo vivo (2).

Entre los géneros implicados con mayor frecuencia en casos de miasis facultativas, se pueden citar Calliphora, Lucilia (= Phaenicia), Phormia y Sarcophaga $(1,2)$. Las enfermedades asociadas y los factores de riesgo descritos con mayor frecuencia para las miasis por estos dípteros son: úlceras expuestas, hemorroides, infecciones bacterianas cutáneas, heridas con abundante exudado, cavidades naturales, falta de aseo personal, ejecución de labores relacionadas con la cría de animales de campo, conductas asociadas al alcoholismo como insensibilidad y costumbre de dormir a la intemperie, ingestión de alimentos contaminados, individuos en abandono con funciones físicas o mentales disminuidas, y pacientes hospitalizados en centros que carecen de hábitos higiénicas o condiciones estructurales adecuadas (2-5).

En Costa Rica, la mayoría de las miasis humanas son de tipo "foruncular" y producidas por la mosca de tórsalo, $D$. hominis (Diptera: Cuterebridae) $(3,4)$. Otras especies de mosca que también se han encontrado provocando miasis humanas en el país son Cochliomyia macellaria y C. hominivorax (4); no obstante, los reportes de miasis humanas por estos dos agentes son cada vez más escasos en Centroamérica.

En la bibliografía médica existe un único reporte de miasis hospitalaria en Costa Rica (3) y hasta la fecha no se han informado casos de miasis hospitalaria por califóridos en el país. Además, este es el primer caso reportado en la literatura científica en Latinoamérica en el que el agente etiológico de la miasis hospitalaria fue Lucilia cuprina, la cual infestó la cavidad nasal de una paciente de edad avanzada y con signos de inmunosupresión.

\section{Caso clínico}

Se trata del caso de una paciente femenina de 91 años que se presentó acompañada de una familiar en el servicio de emergencias de un hospital de referencia en San José, Costa Rica. El motivo de la consulta se debió a un dolor abdominal que irradiaba hacia la cresta ilíaca antero-superior izquierda y que se agudizaba con los cambios de posición. Como antecedentes importantes se encontraron hipertensión arterial de larga data y tos persistente de un mes de duración.

En el momento del examen físico presentaba movilidad de las cuatro extremidades, náuseas, mucosa oral levemente seca, deshidratación de leve a moderada y desnutrición. En la región abdominal se percibió un abdomen blando y doloroso a la palpación en ausencia de masas. La paciente presentaba estabilidad hemodinámica y el Servicio de Ortopedia descartó presencia de fracturas. Las pupilas fueron calificadas como hiporreactivas y la radiografía de tórax reveló un serio compromiso pulmonar con infiltrados bilaterales. Por su condición, se ingresó en el centro hospitalario donde se inició con los estudios de hematología y química sanguínea.

Los resultados de laboratorio demostraron un desequilibrio electrolítico, acidemia y franca leucocitosis con presencia de bandas, lo cual se asoció con un posible cuadro séptico. En virtud de la afectación pulmonar, se instauró asistencia respiratoria mecánica y nutrición entérica a través de una sonda nasogástrica. A partir de ese momento, la salud de la paciente fue en detrimento progresivo.

Al séptimo día luego de su ingreso hospitalario, el personal de enfermería reportó la presencia de larvas provenientes de ambas fosas nasales. En las 24 horas siguientes, se recolectaron cinco de dichas larvas para la determinación de especie y se inició el tratamiento con ivermectina. Según los registros médicos, en los días posteriores no se visualizaron larvas; no obstante, la paciente falleció por una bronconeumonía aguda agravada por insuficiencia renal aguda al doceavo día de internamiento. 
Tres larvas recolectadas fueron fijadas en alcohol al $70 \%$ y referidas al Laboratorio de Entomología Médica de la Facultad de Microbiología de la Universidad de Costa Rica, para la determinación de especie. El material fue disecado y aclarado en lactofenol durante 24 horas, se montó entre lámina y laminilla utilizando medio Hoyer y, posteriormente, se fotografió al microscopio (figura 1).

La observación microscópica de las larvas de tercer estadio permitió establecer las siguientes características: cuerpo cilíndrico con espinas cuticulares unicúspides agrupadas en hileras de dos a siete espinas (figura 1A); al menos, uno de los espiráculos protorácicos con 6 o menos aberturas (figura 1B); esqueleto cefalofaríngeo sin esclerito oral accesorio (figura $1 \mathrm{C}$ ) y peritrema completo que no se proyecta internamente entre las aberturas espiraculares externa y media (figura 1D). Según la clave dicotómica de Thyssen (6), los criterios descritos por Pirali-Kheirabadi, et al. (7), y Nazni, et al. (8), y el análisis taxonómico de las larvas muscomorfas permitió establecer el diagnóstico de L. cuprina como la especie responsable de la miasis. El mismo día en que se evidenciaron las larvas, se recolectaron cinco moscas adultas utilizando redes entomológicas en el exterior del hospital, contiguo al salón donde estuvo internada la paciente. La identificación de estas moscas correspondió a L. cuprina y Musca domestica (9).

\section{Discusión}

En el caso de L. cuprina, el período que transcurre entre la oviposición y el desarrollo larvario hasta el tercer estadio, es de 92 horas, aproximadamente (10). Por lo tanto, el hallazgo de larvas en este estadio supone que la oviposición en los tejidos nasales de la paciente se dio después de la fecha de su ingreso hospitalario. Esto tipifica al presente caso como una miasis hospitalaria. A su vez, este

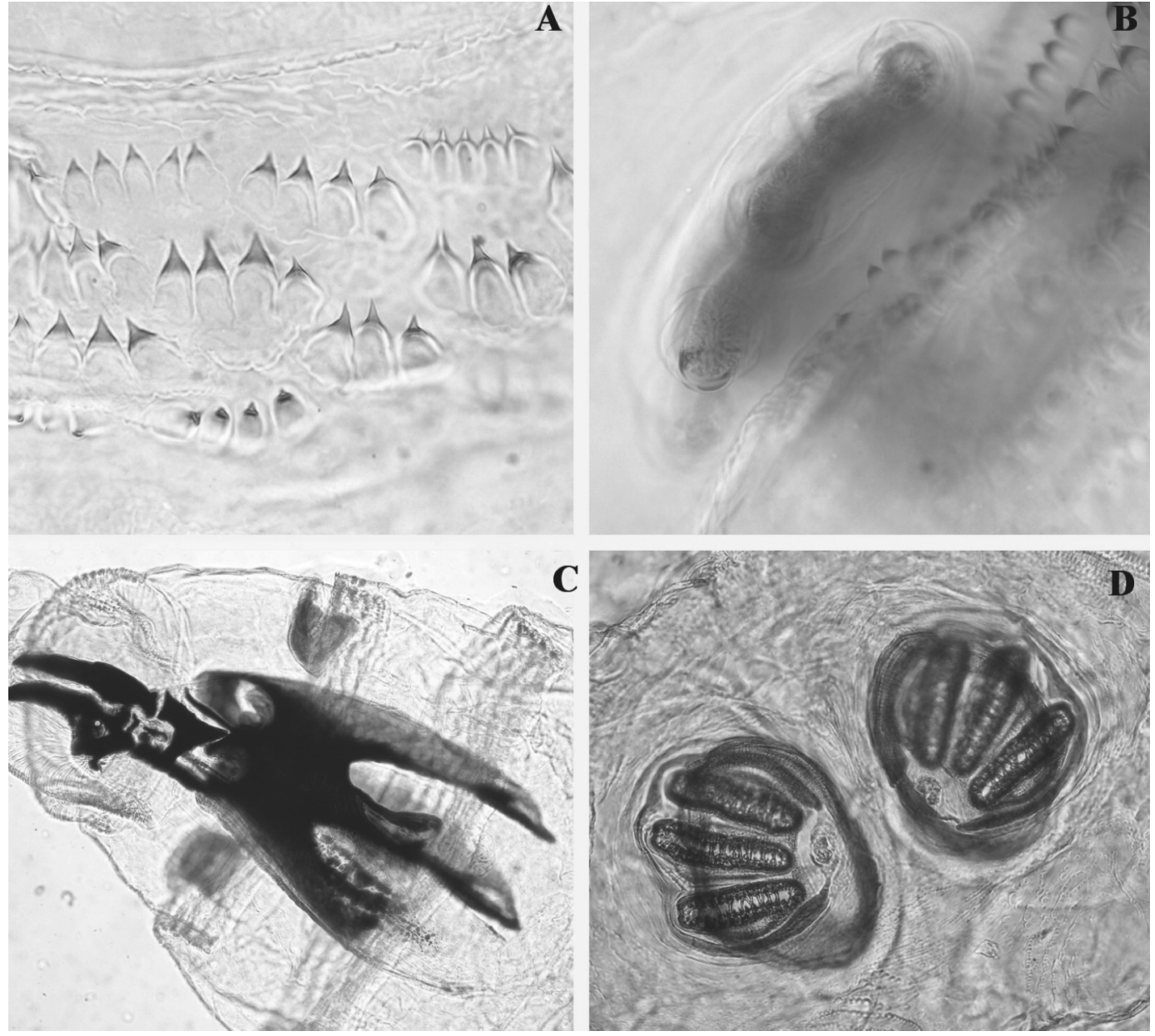

Figura 1. (A) Espinas cuticulares unicúspides (40X). (B) Espiráculo protorácico (40X). (C) Esqueleto cefalo-faríngeo (10X). (D) Espiráculos posteriores (10X). 
sería el primer caso de miasis hospitalaria por $L$. cuprina reportado en Latinoamérica.

Al igual que en la gran mayoría de los informes de miasis $(2,3,5)$, la paciente del presente caso poseía importantes condicionantes de inmunocompromiso como lo fueron la edad avanzada y la desnutrición; además de factores intrínsecamente atrayentes para la oviposición, tales como la presencia de tejidos con abundantes exudados ricos en proteínas que, además, sirven como agentes quimiotácticos para las moscas $(2,3,5,11,12)$. En este caso, los exudados y fluidos corporales expelidos por las aberturas nasales y la cavidad oral, sirvieron como atrayentes para la oviposición en la paciente, provocándole una miasis nasal. Es posible que la mosca responsable haya ingresado a la estructura del hospital por puertas o ventanas cercanas. Esta idea es respaldada por el hallazgo de adultos de L. cuprina en basureros fuera del recinto, pero contiguos a puertas y ventanas del salón donde estuvo internada la paciente. $L$. cuprina es considerada una de las especies de califóridos más frecuentes en Costa Rica, y se ha reportado su tropismo por materia orgánica, fluidos y tejido animal $(13,14)$.

En la bibliografía médica existe un único reporte de miasis hospitalaria en territorio costarricense (3). Dicho caso ocurrió en un paciente de sexo masculino de 86 años de edad que presentaba múltiples complicaciones, respiración asistida y sonda nasogástrica. Transcurridos cinco días de su internamiento, se evidenció la presencia de larvas de mosca en su cavidad oral, que morfológicamente correspondieron a Sarcophaga spp. (Diptera: Sarcophagidae) (3). Por lo tanto, en ambos casos se dieron varios de los factores de riesgo que han sido descritos previamente. Entre estos figuran la acentuada debilidad y el estado de postración de la paciente, la presencia de secreciones o fluidos corporales asociados con la sonda colocada y la presencia de moscas productoras de miasis cerca del entorno hospitalario (2-5).

A pesar de su amplia distribución en el neotrópico (15), L. cuprina nunca antes había sido reportada como agente etiológico de miasis hospitalaria en Costa Rica. En Latinoamérica, tampoco se tiene conocimiento de casos de miasis hospitalaria por L. cuprina. En el campo veterinario, L. cuprina se ha registrado como la mayor causante de miasis cutánea en ovejas, lo que genera grandes pérdidas anuales en la producción (16). Por lo tanto, la ausencia de reportes debe considerarse con precaución en vista de que dicha mosca está ampliamente distribuida en Suramérica, Centroamérica, una parte de México y el Caribe (15). Es muy probable que exista un fenómeno de subregistro de casos.

Finalmente, se debe llamar la atención en cuanto al tratamiento de las miasis. La administración de ivermectina oral se basa en diversa evidencia científica (17-19), además de asistir en el control de las larvas en aquellas miasis cavitarias de difícil acceso $(16,18)$. No obstante, hay que recalcar en la importancia de vigilar y extraer mecánicamente las larvas, dado que podrían invadir el tejido sano y causar daños importantes, entre ellos intenso dolor y deformación (2). Esta posibilidad de invasión, a su vez, depende de la especie de mosca involucrada $(2,4)$.

A pesar de las constantes recomendaciones para mejorar las medidas de asepsia en los pacientes intubados, con úlceras, postrados u con otras condiciones asociadas a miasis, los casos de miasis hospitalaria se continúan reportando alrededor del mundo. Se desea recalcar que no es suficiente extremar las medidas de cuidado e higiene en los pacientes con dichas condiciones, sino que, además, se debe mantener un entorno controlado en cuanto a accesos, manejo de desechos y control de plagas $(9,11)$. La aplicación consciente y responsable de estas medidas favorecen, por un lado, al hospital al cuidar su reputación y, por otro lado y más importante, favorece a los pacientes con factores condicionantes debilitantes e inmunosupresores para no sufrir afecciones ulteriores como lo son las miasis hospitalarias.

\section{Conflicto de intereses}

Los autores no poseen conflictos de intereses que limiten o comprometan la divulgación del presente reporte de caso.

\section{Financiación}

Programa de posgrado de la especialidad en Parasitología Clínica, Universidad de Costa Rica; proyecto ED 548 de la Vicerrectoría de Acción Social.

\section{Referencias}

1. Harwood R, James J. Entomology in human and animal health. 7th edition. London: McMillan Publishing; 1979. p. 548.

2. Hall M, Wall R. Myiasis of humans and domestic animals. Adv Parasitol. 1995;35:257-334. http://dx.doi.org/10.1016/ S0065-308X(08)60073-1 
3. Calderón-Arguedas $\mathrm{O}$, Avendaño A, Vargas-Castro C. Miasis nosocomial por Sarcophaga spp. (Diptera: Sarcophagidae) en un paciente geriátrico de Costa Rica. Rev Costarric Cienc Med. 2004;25:57-61.

4. Calderón-Arguedas $\mathbf{O}$, Sánchez $\mathbf{C}$, Sandí J. Miasis oral por Cochliomyia hominivorax (Diptera: Calliphoridae) en una paciente geriátrica de Costa Rica. Rev Costarric Cienc Med. 2004;16:61-6.

5. Hira PR, Assad RM, Okasha G, Al-Ali FM, Iqbal J, Mutawali KE, et al. Myiasis in Kuwait: Nosocomial infections caused by Lucilia sericata and Megaselia scalaris. Am J Trop Med Hyg. 2004;70:386-9.

6. Thyssen PJ. Keys to identification of immature insects. En: Amendt, Goff ML, Campobasso CP, Grassberger M, editors. Current concepts in forensic entomology. New York: Springer; 2010. p. 25-42.

7. Pirali-Kheirabadi K, Cheraghchi-Bashi M, Navidpour $\mathbf{S}$. Infestation of an owl (Bubo bubo) with Lucilia spp. Comp Clin Pathol. 2010;19:221-4. http://dx.doi.org/10.1007/ s00580-009-0838-3

8. Nazni WA, Jeffery J, Lee HL, Lailatu-Amar MN, Heo CC, Sadiyah I, et al. Nosocomial nasal myiasis in an intensive care unit. Malaysian J Pathol. 2011;33:53-6.

9. Barros de Carvalho CJ, Antunes de Mello-Patiu CA. Key to the adults of the most common forensic species of Diptera in South America. Rev Bras Entomol. 2008;52:390 406. http://dx.doi.org/10.1590/S0085-56262008000300012

10. O'Flynn M A. The succession and rate of development of blowflies in carrion in southern Queensland and the application of these data to forensic entomology. J Aust Entomol Soc. 1983;22:137-48. http://dx.doi.org/10.1111/ j.1440-6055.1983.tb01860.x

11. Sherman RA, Roselle G, Bills C, Danko LH, Eldridge N. Healthcare-associated myiasis: Prevention and intervention.
Infect Control Hosp Epidemiol. 2005;26:828-32. http:// dx.doi.org/10.1086/502501

12. Beckendorf R, Klotz SA, Hinkle N, Bartholomew W. Nasal myiasis in an intensive care unit linked to hospitalwide mouse infestation. Arch Intern Med. 2002;162:63840. http://dx.doi.org/10-1001/pubs.Arch Intern Med.-ISSN0003-9926-162-6-isa10010.

13. Calderón-Arguedas $\mathbf{O}$, Troyo A, Avendaño A. Vejigas animales como sitios de oviposición y desarrollo de moscas en festividades, Heredia - Costa Rica. Rev Costarric Salud Pública. 2008;17:15-8.

14. Jirón LF. Sobre moscas califóridas de Costa Rica (Diptera: Cyclorrhapha). Brenesia. 1979;16:221-3.

15. Pape T, Wolff M, Amat E. Los califóridos, oéstridos, rinofóridos y sarcofágidos (Diptera: Calliphoridae, Oestridae, Rhinophoridae, Sarcophagidae) de Colombia. Biota Colombiana. 2004;5:201-8.

16. Norris KR. The bionomics of blow flies. Annu Rev Entomol. 1965;10:47-68. http://dx.doi.org/10.1146/annurev. en.10.010165.000403

17. De Hollanda-Ramírez AM, Silva-Rodríguez AR, Zaracho G. Ivermectina en el tratamiento de la miasis humana. An Fac Cienc Méd. 2005;38:62-71.

18. Shinohara EH, Martini MZ, de Oliveira-Neto HG, Takahashi A. Oral myiasis treated with ivermectin: Case report. Braz Dent J. 2004;15:79-81. http://dx.doi. org/10.1590/S0103-64402004000100015

19. Ribeiro FA, Pereira CS, Alves A, Marcon MA. Tratamento da mí́ase humana cavitária com ivermectina oral. Rev Bras Otorrinolaringol. 2001;67:755-61. http://dx.doi.org/10.1590/ S0034-72992001000600002 